\title{
A first XMM-Newton look at the relativistic double pulsar PSR J0737-3039
}

\author{
A. Pellizzoni, A. De Luca, S. Mereghetti, A. Tiengo, F. Mattana, P. Caraveo \\ Istituto di Astrofisica Spaziale e Fisica Cosmica, \\ Sezione di Milano "G.Occhialini" - CNR \\ v.Bassini 15, I-20133 Milano, Italy \\ alberto@mi.iasf.cnr.it \\ M. Tavani \\ Istituto di Astrofisica Spaziale e Fisica Cosmica, \\ Sezione di Roma - CNR \\ v.Fosso del Cavaliere 100, I-00133 Roma, Italy \\ G.F. Bignami \\ Centre d'Étude Spatiale des Rayonnements, CNRS-UPS, \\ 9, avenue du Colonel Roche, 31028 Toulouse Cedex 4, France \\ and Università degli Studi di Pavia, Dipartimento di Fisica Nucleare e Teorica, \\ v.Bassi 6, I-27100 Pavia, Italy
}

\begin{abstract}
We present the results of a $50 \mathrm{ks}$ long $\mathrm{X}$-ray observation of the relativistic double pulsar system PSR J0737-3039 obtained with the XMM-Newton satellite in March 2004. The source has a soft spectrum (power law photon index $=$ $3.5_{-0.3}^{+0.5}$ ) and a $0.2-10 \mathrm{keV}$ luminosity of $\sim 3 \times 10^{30} \mathrm{erg} \mathrm{s}^{-1}$ (assuming a distance of $500 \mathrm{pc}$ ), consistent with the values derived from a previous Chandra observation. No flux variations have been detected, implying the absence of large orbital modulations. The high time resolution of the EPIC instrument has allowed us to perform the first search for X-ray pulsations from this system. The result was negative, with an upper limit of $60 \%$ on the pulsed fraction of the $22 \mathrm{~ms}$ pulsar.
\end{abstract}

Subject headings: stars: neutron $-\mathrm{X}$-rays: stars 


\section{Introduction}

The short-period double radio pulsar system PSR J0737-3039 (Burgay et al. 2003; Lyne et al. 2004) is of paramount interest as a probe for theories of strong field gravity and represents a unique laboratory for the study of the magnetospheres of radio pulsars. The system consists of a fast, recycled radio pulsar (PSR A, P=22.7 ms) orbiting its slower companion (PSR B, P=2.77 s) with an orbital period of only 2.4 hours. The fact that we observe it nearly edge-on (inclination $i=87^{\circ}$ ), provides an additional tool to further constrain the magnetospheric structures by reciprocal occultation of the two pulsars and their shocked winds near conjunction.

Due to the interaction of the relativistic wind of the fast spinning pulsar A $\left(\dot{E}_{\text {rot }}^{A}=5.8 \times 10^{33}\right.$ erg s$\left.{ }^{-1}\right)$ with the magnetosphere of its much less energetic companion $\left(\dot{E}_{\text {rot }}^{B}=1.6 \times 10^{30} \mathrm{erg}\right.$ $\mathrm{s}^{-1}$ ), the formation of a bow-shock, likely emitting at high energies, is expected. The predicted fluxes are roughly comparable to those expected for magnetospheric and/or surface emission from the pulsars. Alternately, most of the high-energy emission could arise from the interactions between the pulsar relativistic winds and the interstellar medium. Detailed emission models and geometry are clearly speculative without valuable high-energy observations, particularly at $\mathrm{X}$-ray energies.

PSR J0737-3039 was first detected in the 0.2-10 keV range during a short (10 ks) Chandra observation (McLaughlin et al. 2004), with an X-ray luminosity of $\mathrm{L}_{x}=2 \times$ $10^{30}(\mathrm{~d} / 0.5 \mathrm{kpc})^{2} \mathrm{erg} \mathrm{s}^{-1}$, roughly equal to the entire spin-down luminosity of the slow pulsar $\mathrm{B}$ and corresponding to only a small fraction of the spin-down luminosity of pulsar A. With the detection of $\sim 80$ pulsar photons spanning one orbital period, the Chandra data could only poorly constrain the source spectrum and were of limited use for the study of variability. Furthermore the time resolution of the ACIS instrument was not enough to search for pulsations at the spin periods of the two pulsars.

In this letter we present the analysis of a $50 \mathrm{ks}$ long XMM-Newton observation of PSR J0737-3039 performed through the Director's Discretionary Time program and made publicly available. Although episodes of high particle background affected this observation, these data yield an improvement in statistics by a factor $\sim 10$ and have allowed us to carry out the first search for fast pulsations from this system. In addition, the coverage of $\sim 5$ orbits allows to set limits on the orbital variability of the $\mathrm{X}$-ray emission. 


\section{XMM-Newton observation and data reduction}

The XMM-Newton observation started on 2004, March 28 at 07:47:26 UT and lasted $\sim 51 \mathrm{ks}$. We report here on data collected by the EPIC instrument, which consists of a pn CCD camera (Strüder et al. 2001) and of two MOS CCD detectors (Turner et al. 2001), for a total total collecting area of $\sim 2500 \mathrm{~cm}^{2}$ at $1.5 \mathrm{keV}$. The pn camera was operated in "Fast Timing" mode, with a time resolution of $0.03 \mathrm{~ms}$, achieved at the cost of the loss of positional information along the detectors columns and of a higher background. The MOS1 camera was operated in "Full Frame" mode (2.6 s time resolution, imaging across the full $15^{\prime}$ radius telescope field of view), while the MOS2 was set in "Small Window" mode (0.3 s time resolution, imaging on $\mathrm{a} \sim 1^{\prime} \times 1^{\prime}$ portion of the central $\left.\mathrm{CCD}^{1}\right)$. The Observation Data Files (ODFs) were retrieved through the XMM-Newton Science archive. The data reduction was performed using the most recent release of the XMM-Newton Science Analysis software (SASv6.0.0), with the standard pipeline tasks (epproc for pn data and emproc for MOS data). PSR J0737-3039 is a faint X-ray source with a flux of a few $10^{-14} \mathrm{erg} \mathrm{cm}^{-2} \mathrm{~s}^{-1}$ and therefore a careful study of the instrumental background is required. Two different issues are of particular relevance:

- Soft flares in pn data. These are due to the interaction of high-energy particles with the CCD; they are seen as short (0.1-0.5 s) and intense (up to thousands of counts $\mathrm{s}^{-1}$ ) bursts of events, with a typical energy of $\sim 0.22 \mathrm{keV}$ (mono-pixel events) and $\sim 0.45$ $\mathrm{keV}$ (bi-pixel events) and with a highly anisotropic distribution across the CCD, which prevents standard subtraction. Following Burwitz et al. (2004), we used an ad hoc Good Time Interval (GTI) filter to remove such background component. This was done by intersecting two independent GTI files which were built by setting a threshold of $15 \mathrm{cts} \mathrm{s}^{-1}$ on boxcar-smoothed light curves ( $0.1 \mathrm{~s}$ time bins) extracted in the $0.2-0.3$ $\mathrm{keV}$ range for mono-pixel events and in the $0.4-0.5 \mathrm{keV}$ range for bi-pixel events. The rejected frames amount to $\sim 5400 \mathrm{~s}(\sim 11 \%$ of the observing time). As a result, the soft flare background component is almost totally suppressed.

- Soft proton flares. The whole observation is affected by medium-intensity proton flares. This is a point of concern especially for the pn camera, since in Fast Timing mode the collapse of data along detector columns increases the background count rate in the source extraction region by a factor $\sim 40$ wrt the standard imaging modes. In the MOS cameras, taking advantage of 2-D spatial resolution, a less stringent GTI filtering is required. GTI files were built for each of the three cameras in order to reject the

\footnotetext{
${ }^{1}$ Peripheral CCDs are read with a $2.6 \mathrm{~s}$ frame time as in Full Frame mode
} 
time intervals most affected by soft protons (for MOS cameras we set thresholds of 800 cts/bin on $40 \mathrm{~s}$ bin light curves extracted from peripherals CCDs in 0.2-12 keV; for the pn of $140 \mathrm{cts} / \mathrm{bin}$ on a $40 \mathrm{~s}$ bin light curve, 0.5-12 keV range).

The cleaned event lists correspond to dead-time corrected exposures of $20450 \mathrm{~s}, 36900 \mathrm{~s}$ and $33700 \mathrm{~s}$ for the pn, MOS1 and MOS2 respectively. The target was detected with a 0.2-3 $\mathrm{keV}$ net count rate of $(2.9 \pm 0.3) \times 10^{-2} \operatorname{cts~s}^{-1},(3.9 \pm 0.4) \times 10^{-3} \operatorname{cts~s}^{-1}$ and $(3.7 \pm 0.5) \times 10^{-3}$ cts $\mathrm{s}^{-1}$, in the pn, MOS1 and MOS2. These count rates were estimated using a $15^{\prime \prime}$ radius region (enclosing $>65 \%$ of the source counts) in each of the MOS cameras, and a 8 pixel wide strip (4.1" pixel size) in the pn.

\section{Spectral analysis}

The source spectrum was extracted from the regions described above, and appropriate background regions were selected on the same CCD chip where the target is imaged. Spectra were binned in order to have at least 25 counts per channel for the MOSs, 80 counts per channel in the pn. Ad hoc response matrices and effective area files were generated with the SAS tasks rmfgen and arfgen. The spectral analysis was performed using XSPEC v11.3. Due to the very soft spectrum of PSR J0737-3039, the background dominates above 2.5 $\mathrm{keV}$. Therefore we restricted the analysis to the $0.2-3 \mathrm{keV}$ range, which results in 160 MOS1 counts, 130 MOS2 counts, and 2910 pn counts. We estimate that the background accounts for $\sim 20 \%$ of the above values in the case of the two MOS and for $\sim 80 \%$ in the pn.

The spectra from MOS1, MOS2 and pn were fitted simultaneously. An absorbed power law model with photon index $\Gamma=3.5_{-0.3}^{+0.5}$ and absorption $\mathrm{N}_{H}=(7.0 \pm 2.5) \times 10^{20} \mathrm{~cm}^{-2}$, describes well the data ${ }^{2}$. A confidence contour plot for the spectral parameters $\mathrm{N}_{H}$ and $\Gamma$ is presented in Fig.1. A photon index of 2 is ruled out at more than $99.99 \%$ level. Assuming a distance of $500 \mathrm{pc}$, the inferred $0.2-10 \mathrm{keV}$ luminosity is $\sim 3.4 \times 10^{30} \mathrm{erg} \mathrm{s}^{-1}$. An equally good fit is obtained with a thermal bremsstrahlung model, with temperature of $0.5 \pm 0.1$ $\mathrm{keV}$ and $\mathrm{N}_{H}<5 \times 10^{20} \mathrm{~cm}^{-2}$. Using a blackbody model the fit is somewhat worse, with a best fit temperature of $0.16 \pm 0.01 \mathrm{keV}$ and a lower interstellar absorption $\left(\mathrm{N}_{H}<1.5 \times 10^{20}\right.$ $\mathrm{cm}^{-2}$ ). All the spectral results are summarized in Table 1 . We tried also a composite model consisting of a blackbody plus a power law with photon index fixed at 2 . The quality of the fit did not improve compared to the best single-component models. The $0.2-10 \mathrm{keV}$ flux of a possible hard non-thermal spectral component is $\sim 2.5 \times 10^{-14} \mathrm{erg} \mathrm{cm}^{-2} \mathrm{~s}^{-1}$, corresponding

\footnotetext{
${ }^{2}$ All the reported errors on the spectral parameters are at $90 \%$ confidence for a single interesting parameter
} 
to a luminosity of $\sim 7 \times 10^{29} \mathrm{erg} \mathrm{s}^{-1}$.

\section{Timing analysis}

As mentioned above, a relatively high and time variable background affected this observation. Therefore to search for orbital modulations in PSR J0737-3039 we first computed background subtracted light curves in $10 \mathrm{~s}$ bins for the MOS1 and MOS2. For the source extraction we used a circle of radius $17^{\prime \prime}$, while the background was estimated from much larger source free regions in the same CCD chips. The resulting $0.3-2 \mathrm{keV}$ light curves were then folded at the orbital period (2.45 hours), trying different number of bins and also varying the phases of the bin boundaries. We show in Fig.2 the light curves for the MOS1, MOS2 and their sum folded in 20 bins, as an example. No significant evidence for variability was found, also repeating the analysis with more stringent data cleaning selections. Considering that the adopted extraction region contains 557 counts $(\sim 185$ of which we estimate are due to the background), a $99 \%$ confidence level upper limit of the order of $40 \%$ can be set on the pulsed fraction in the simplest hypothesis of a sinusoidal modulation.

For the search of pulsations at the spin period of pulsar A we used the pn data in the 0.3-2 keV range extracted from time intervals with low particle background. This resulted in a net exposure of $13800 \mathrm{sec}$, yielding 850 counts ( $\sim 62 \%$ of which from the background). The times of arrival were converted to the Solar system barycenter and we also corrected them for the orbital motion and relativistic effects of the binary system according to Blandford

\& Teukolsky (1976). This was done with a program we wrote ad hoc and tested using a XMM-Newton observation of the binary millisecond pulsar XTE J1751-30 (Miller et al. 2003).

Figure 3 shows the pn data folded at the expected period of pulsar A, based on the ephemerids reported by Lyne et al. (2004). The light curve is consistent with a constant flux. In the assumption of a sinusoidal modulation we can set a $99 \%$ c.l. upper limit of $\sim 60 \%$ on the pulsed flux from pulsar A.

Finally, we also searched for periodic modulations at the period of pulsar B using both the pn and MOS2 data with negative results ( $~ 60 \%$ upper limit).

\section{Discussion}

Rotation-powered pulsars emit of the order of $10^{-3} \dot{E}_{\text {rot }}$ in soft X-rays (Becker \& Trümper 1997; Possenti et al. 2002). This implies that the expected luminosity for pul- 
sar A should be of the order of $10^{31} \mathrm{erg} \mathrm{s}^{-1}$ while B should be undetectable. With a pure power-law fit of the data, the efficiency for conversion of rotational energy of A to X-rays is $6 \times 10^{-4}(\mathrm{~d} / 0.5 \mathrm{kpc})^{2}$ a value comparable with the Chandra result and compatible (taking into account a factor of $\sim 2$ distance uncertainty) with the emission originating solely from the magnetosphere of pulsar A.

The very soft observed X-ray spectrum $(\Gamma=3.5)$ is not unheard of when considering the class of recycled pulsars. Such sources are known to show broadly different spectral phenomenologies (see e.g. Becker \& Aschenbach 2002 and references therein; Webb et al. 2004a,b), ranging from very hard, non-thermal spectra with power law photon index $\Gamma<$ 2 (e.g. PSR B1821-24, PSR B1937-21), to very soft $(\Gamma \sim 3-4)$ and possibly thermal spectra (e.g. PSR J0030+0451, PSR J2124-3358). Indeed, PSR J0437-4715, the nearest and brightest ms pulsar, was already known in the ROSAT era to have a composite spectrum showing both a soft and a hard component (Zavlin \& Pavlov 1998). Thus, an interpretation of the observed X-ray emission from the PSR J0737-3039 system as "normal" recycled pulsar radiation originating from pulsar A seems the simplest one. The upper limit on the pulsed fraction at the 22 ms period $(\sim 60 \%)$ is not inconsistent with this picture, since modulations at the $\sim 30-40 \%$ level have been observed in some cases.

However, the PSR J0737-3039 system offers other possible sources of high-energy photons with comparable power than magnetospheric emission from A. A remarkable one might be the bow-shock that forms near pulsar B owing to the collision between A's relativistic wind and B's magnetosphere (Arons et al. 2004).

If the magnetisation of A's wind is low, the shock is expected to be strongly dissipative and a large fraction of the incoming energy flux may be radiated in X-rays. Within distance uncertainty, the observed luminosity of $\sim 3 \times 10^{30}(\mathrm{~d} / 0.5 \mathrm{kpc})^{2} \mathrm{erg} \mathrm{s}^{-1}$ is compatible with a fraction $(\leq 50 \%)$ of the maximum $\mathrm{X}$-ray flux expected from the bow-shock. This limit is set by $\mathrm{L}_{\max } \sim \dot{E}_{A} \Delta \Omega / 4 \pi \sim 3 \times 10^{31} \mathrm{erg} \mathrm{s}^{-1}$, where $\Delta \Omega \sim 6 \times 10^{-2}$ sr (Lyutikov 2004) is the solid angle seen from pulsar A enclosing the magnetosheath around pulsar B.

As already seen with Chandra, the X-ray photon index is rather steep, at variance with the flat $\nu \mathrm{F}_{\nu}$ spectrum typically expected from shock acceleration. This is independently confirmed by our data which give a range of allowed slopes even less compatible with the "canonical" shock value $\Gamma \sim 2$ (see Fig.1). Such a soft spectrum could be due to the presence of an unusual low-energy relativistic electron population as required in the magnetosheath model (Arons et al. 2004; Lyutikov 2004) to provide A and B's eclipses by synchrotron absorption.

Within the bow-shock/magnetosheath model, one might expect a flux modulation as a 
function of the orbital period owing to the changing view of the shock front. In particular, the shocked wind is expected to flow away from the head of the bow-shock in a direction roughly parallel to the shock (Lyutikov 2004; Granot \& Meszaros 2004). This might imply relativistic beaming of the radiation emitted by the shocked plasma, resulting in a $\lesssim 50 \%$ modulation of the observed emission as a function of the orbital phase with possible peaks at 90 degrees from conjunctions. Our analysis of the flux variations as a function of the orbital phase allow us to exclude the presence of any large orbital modulation $\geq 40 \%$. However, we note that a low significance peak (MOS1 data) is seen in the orbital light curve shifted roughly 0.3 in phase from inferior conjunction.

It is worth considering also possible modulation of the $\mathrm{X}$-ray flux as a function of the spin period of pulsar B $(2.77 \mathrm{~s})$. The rotation of pulsar B, assuming a misalignement of its magnetic pole relative to its spin axis (Ramachandran et al. 2004), would change the magnetic pressure behind the bow-shock as well as the values of the physical quantities of the shocked plasma with a periodicity equal to the spin period. No periodicity with $\mathrm{P}_{B}$ was detected in pn and MOS data.

Although not improving the fit to the data with respect to one-component models, the scenarios involving a non-thermal component, originating from A's magnetosphere and/or from a bow-shock, superimposed to a thermal one is still an intriguing possibility. The luminosity of the non-thermal component we derived assuming a power-law with canonical shock acceleration photon index $\sim 2$ is nearly an order of magnitude smaller than that expected from the bow-shock/magnetosheath model (Granot \& Meszaros 2004). These authors suggest that the likeliest explanation for the $\mathrm{X}$-ray emission is the pulsar A wind just behind the bow-shock caused by the systemic motion in the ISM. This model provides luminosities of $\sim 6 \times 10^{29} \mathrm{erg} \mathrm{s}^{-1}$, a value in agreement with our results for the composite BB+PL fit. In this case, little or no modulation with the orbital period, $\mathrm{P}_{A}$ and $\mathrm{P}_{B}$ is expected.

The remarkable steepness of the spectrum might be related to blackbody emission with temperature $\sim 0.15 \mathrm{keV}$ and luminosity roughly half of the total X-ray luminosity. Thermal emission from polar caps could still play a role for pulsar A, despite its large spin-down age ( $\sim 210$ Myrs). Furthermore, it is in principle conceivable that a portion of A's relativistic Poynting and particle flux might heat the surface of $\mathrm{B}$, giving rise to thermal radiation (Zhang \& Loeb 2004). To match both luminosity and black-body temperature, one could consider the possibility that part of A's wind energy is absorbed by B's magnetosphere and driven towards $B$ surface. If the expected energy input from $A\left(\sim 10^{31} \mathrm{erg} \mathrm{s}^{-1}\right)$ could be transferred with an efficiency $\geq 10 \%$, at B's surface, the resulting heating could match the thermal luminosity of $\sim 10^{30} \mathrm{erg} \mathrm{s}^{-1}$ obtained from our spectral fits. There is no way to obtain high temperatures $(>1 \mathrm{eV})$ directly from the heating of structures with magnetospheric size. 
Future high-energy observations of PSR J0737-3039 could better trade spectral models and reduce upper limits on orbital modulation and pulsed fractions from A and B providing conclusive evidence of the magnetospheric, thermal or bow-shock origin of the X-ray flux. For example, another $\sim 50$ ks XMM-Newton /EPIC observation with pn operating in "Small Window" mode, trading sensitivity vs. time resolution, could strongly improve the search for $\mathrm{P}_{\text {orb }}$ and $\mathrm{P}_{B}$ modulations as well as spectral fits, while further observations with pn in "Fast Timing" mode, looking for A's pulsations, should be much longer if aimed to detect the small pulsed fraction in principle expected from a relatively weak millisecond pulsar.

Based on observations with XMM-Newton, an ESA science mission with instruments and contributions directly funded by ESA member states and the USA (NASA). We thank Norbert Schartel for approving this observation within the frame of discretionary time of Project Scientist. 
Table 1: Results of the spectral analysis. The quoted errors are at $90 \%$ confidence for a single interesting parameter. The errors on fluxes are at $1 \sigma$ level.

\begin{tabular}{lcccc}
\hline \hline & Power Law & Bremsstrahlung & Blackbody & BB + PL \\
\hline$\chi_{\nu}^{2} /$ d.o.f. & $0.92 / 40$ & $0.86 / 40$ & $1.11 / 40$ & $0.90 / 39$ \\
\hline $\mathrm{N}_{H}\left(\mathrm{~cm}^{-2}\right)$ & $7.0 \pm 2.5$ & $<5$ & $<1.5$ & $<4$ \\
\hline$\alpha_{p h}$ & $3.5_{-0.3}^{+0.5}$ & - & - & 2 (fixed) \\
\hline $\mathrm{kT}(\mathrm{keV})$ & - & $0.5 \pm 0.1$ & $0.15 \pm 0.01$ & $0.14 \pm 0.01$ \\
\hline $\mathrm{F}_{0.2-3 \mathrm{keV}}^{(a)}\left(10^{-14} \mathrm{erg} \mathrm{cm}^{-2} \mathrm{~s}^{-1}\right)$ & $3.7 \pm 0.5$ & $4.1 \pm 0.5$ & $3.3 \pm 0.5$ & $4.0 \pm 0.5$ \\
\hline $\mathrm{L}_{0.2-10 \mathrm{keV}}^{(b)}\left(\mathrm{erg} \mathrm{s}^{-1}\right)$ & $3.0 \times 10^{30}$ & $1.1 \times 10^{30}$ & - & $6.9 \times 10^{29}$ \\
\hline $\mathrm{L}_{B B}^{(c)}\left(\mathrm{erg} \mathrm{s}^{-1}\right)$ & - & - & $9.8 \times 10^{29}$ & $7.1 \times 10^{29}$ \\
\hline
\end{tabular}

${ }^{a}$ Observed flux in the $0.2-3 \mathrm{keV}$ range.

${ }^{b} 0.2-10 \mathrm{keV}$ luminosity (excluding BB component) for a distance of $500 \mathrm{pc}$.

${ }^{c}$ bolometric luminosity for $\mathrm{BB}$ component for a distance of $500 \mathrm{pc}$. 


\section{REFERENCES}

Arons, J., Backer, D.C., Spitkovsky, A., \& Kaspi, V.M. 2004, to Appear in Proceedings of the 2004 Aspen Winter Conf. on Astrophysics 'Binary Radio Pulsars', ASP Conf.Series, (eds.) F. Rasio \& I. Stairs (astro-ph/0404159)

Becker, W., \& Aschenbach, B. 2002, Proceedings of the 270. WE-Heraeus Seminar on Neutron Stars, Pulsars, and Supernova Remnants. MPE Report 278, (eds.) W. Becker, H. Lesch, and J. Trümper. Garching bei München: Max-Plank-Institut für extraterrestrische Physik, p.64

Becker, W., \& Trümper, J. 1997, A\&A, 326, 682

Blandford, R., \& Teukolsky, S.A. 1976, ApJ, 205, 580

Burgay, M. et al. 2003, Nature, 426, 531

Burwitz, V. et al. 2004, SPIE, 5165, 123

Granot, J., \& Meszaros, P. 2004, 609, L17

Lyne, A.G. et al. 2004, Science, 303, 1153

Lyutikov, M. 2004, MNRAS, submitted (astro-ph/0403076)

McLaughlin, M.A. et al. 2004, ApJ, 605, L41

Miller, J.M. et al. 2003, ApJ, 583, L99

Possenti, A., Cerutti, R., Colpi, M., \& Mereghetti, S. 2002, A\&A, 387, 993

Ramachandran, R. et al. 2004, ApJ, submitted (astro-ph/0404392)

Strüder, L. et al. 2001, A\&A, 365, L18

Turner, M.J.L. et al. 2001, A\&A, 365, L27

Webb, N.A., Olive, J.F., \& Barret, D. 2004a, A\&A, 417, 181

Webb, N.A. et al. 2004b, A\&A, 419, 269

Zhang, B., \& Loeb, A. 2004, ApJ, submitted (astro-ph/0406609)

Zavlin, V.E., Pavlov, G.G. 1998, A\&A, 329, 583 
Fig. 1.- Contour plot of spectral parameters for the absorbed power law model. The plotted confidence levels are $68.3 \%, 90 \%$ and $99 \%$.

Fig. 2.- Background subtracted light curves folded at the orbital period (8834.5350432 s). MOS1 (top), MOS2 (middle) and MOS1+MOS2 (bottom). The corresponding $\chi^{2}$ values are 17.9 (MOS1), 10.8 (MOS2) and 24.3 (MOS1+MOS2) for 19 degrees of freedom. The dashed line marks the inferior conjunction of $\mathrm{B}$ (phase $=0.43$ ) when the two stars are at their closest on the sky and B is nearer to the Earth.

Fig. 3. - PN data folded at the period ( $\mathrm{P}=22.6993786 \mathrm{~ms}$ ) expected for pulsar $\mathrm{A}$. The dashed line indicates the estimated background contribution which has not been subtracted since it does not vary on this short time scale. 


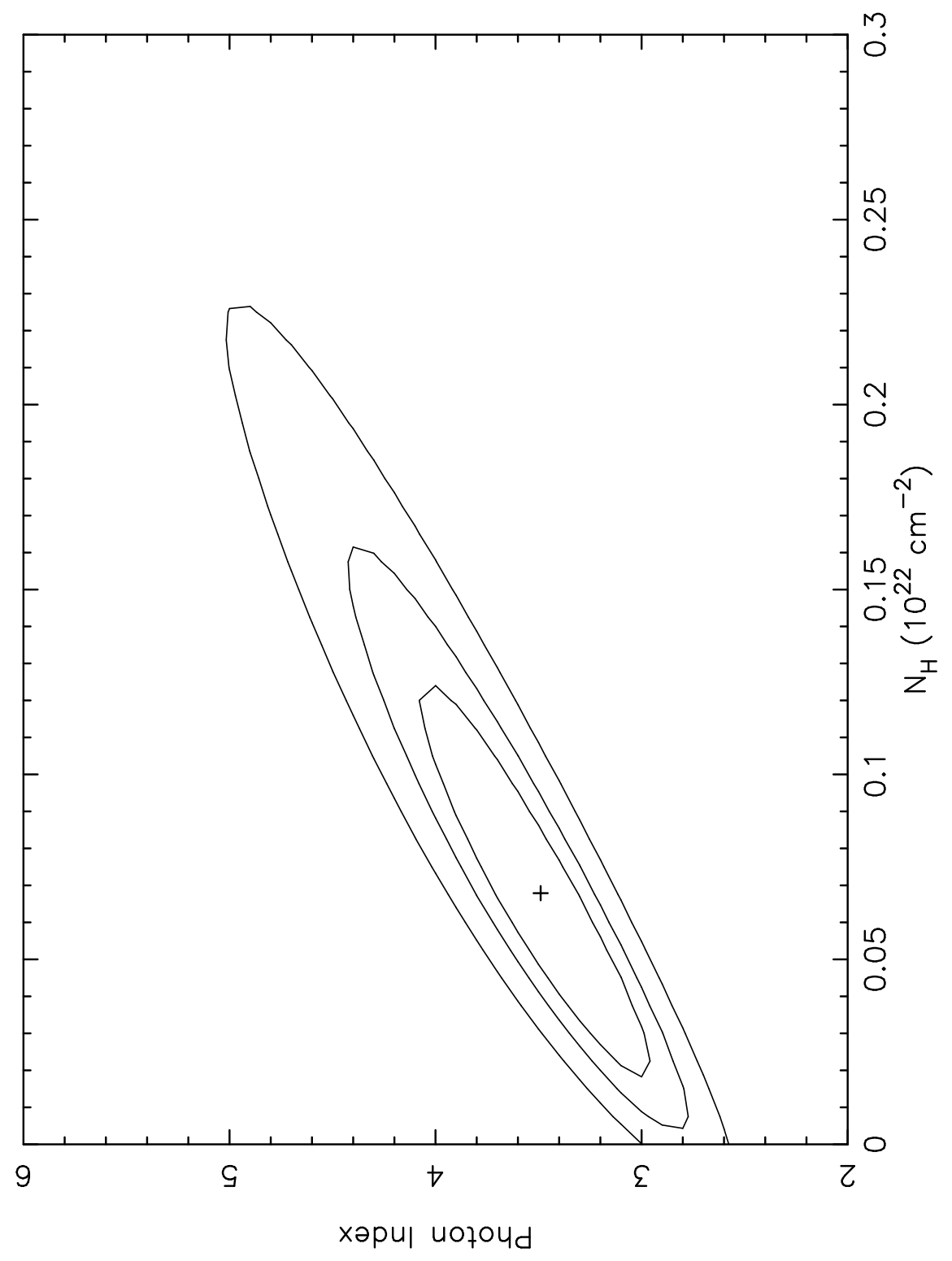




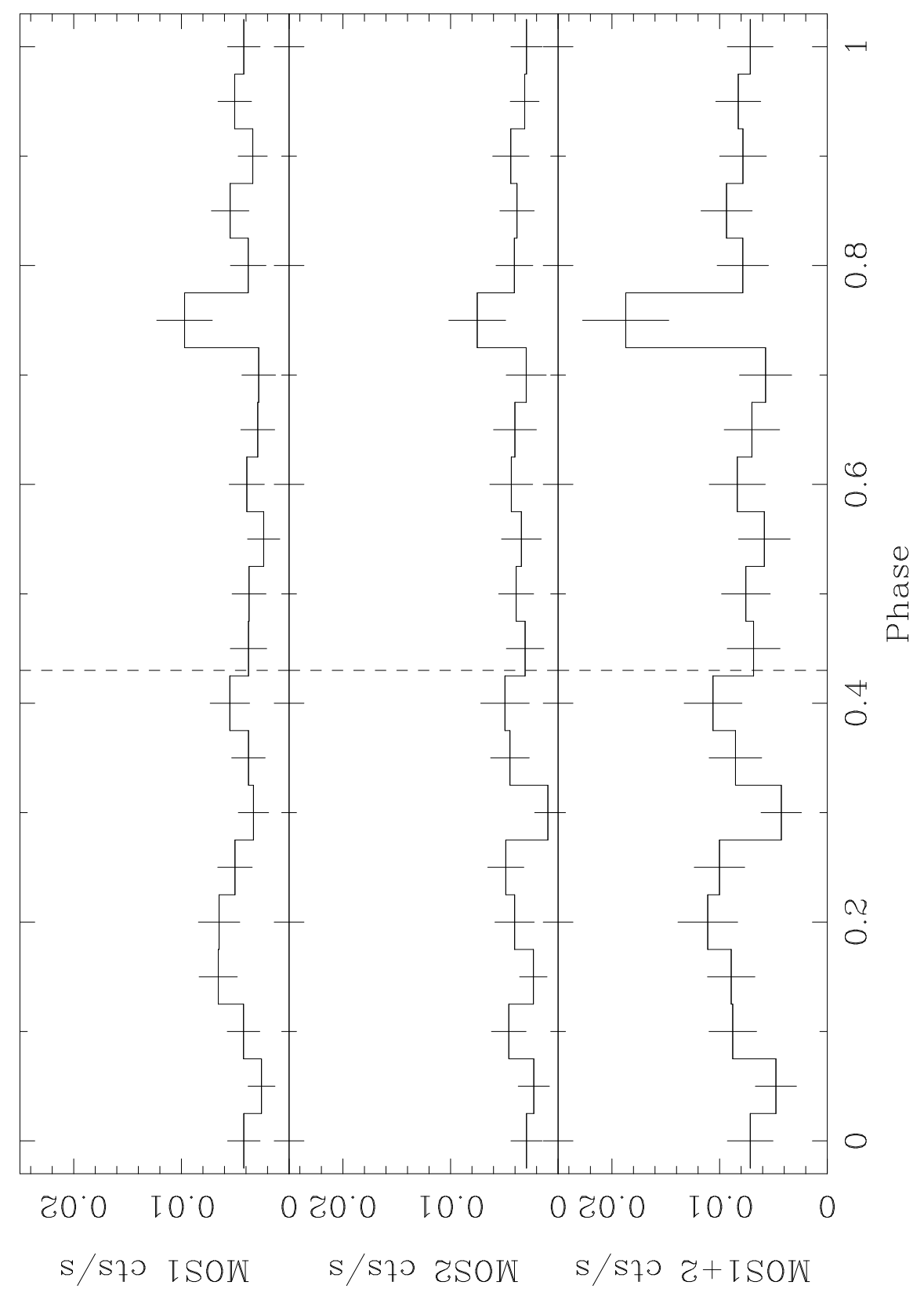


- $14-$

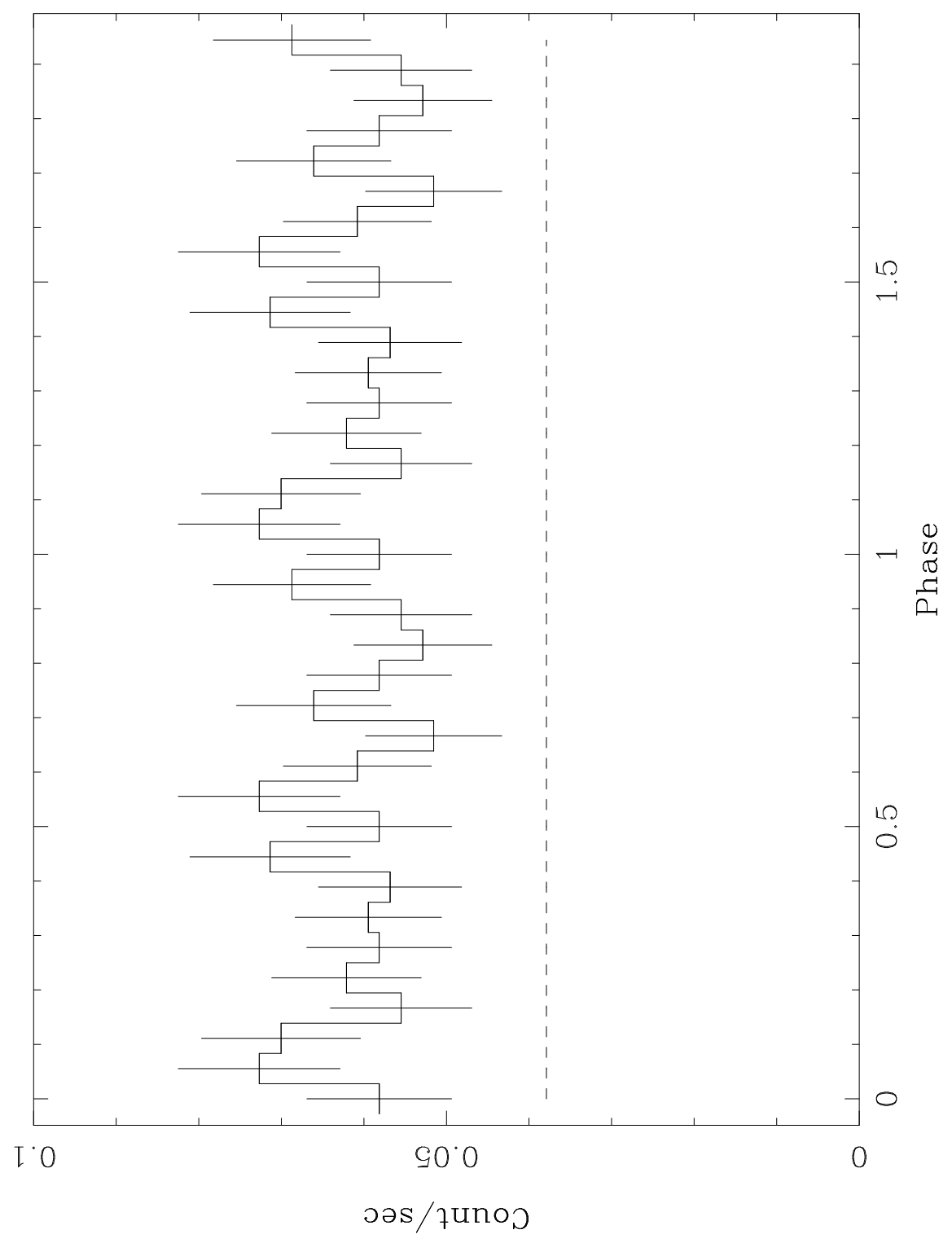

